\title{
Les conseils de quartier, un révélateur des difficultés d'émergence du nouveau " pouvoir consultatif »
}

Philippe Breton et Célia Gissinger

\section{(2) OpenEdition}

\section{Journals}

Édition électronique

URL : https://journals.openedition.org/communicationorganisation/773

DOI : 10.4000/communicationorganisation. 773

ISSN : 1775-3546

Éditeur

Presses universitaires de Bordeaux

Édition imprimée

Date de publication : 1 juin 2009

Pagination : 124-135

ISSN : 1168-5549

Référence électronique

Philippe Breton et Célia Gissinger, «Les conseils de quartier, un révélateur des difficultés d'émergence du nouveau " pouvoir consultatif » », Communication et organisation [En ligne], 35 | 2009, mis en ligne le 01 décembre 2012, consulté le 28 juin 2022. URL : http://journals.openedition.org/

communicationorganisation/773 ; DOI : https://doi.org/10.4000/communicationorganisation.773 
Dossier : Repenser la communication des organisations publiques

\begin{abstract}
Résumé
Les « conseils de quartier », devenus obligatoires depuis la loi de 2002 pour les communes de plus de 80000 habitants, témoignent de l'émergence d'un «pouvoir consultatif» aux côtés des pouvoirs législatif, exécutif, judiciaire. La problématique de la séparation des pouvoirs est au cœur de cet article. L'analyse porte sur trois éléments clefs: le pouvoir consultatif est démocratiquement nécessaire, techniquement possible et politiquement difficile à mettre en place. Elle s'appuie sur une observation de terrain faite entre les mois de mai à juillet 2008, sur les conseils de quartier mis en place jusque là par la Ville de Strasbourg.
\end{abstract}

\title{
Mots-clés
}

Pouvoir consultatif, conseils de quartier, démocratie.

\section{Abstract}

The neighborhood councils, become mandatory since the 2002 law for municipalities of more than 80000 people, reflect the emergence of a "consultative power " alongside the legislative, executive, judicial. The issue of separation of powers is at the heart of this article. The analysis focuses on three key elements: the advisory power is democratically necessary, technically feasible and politically difficult to implement.

\section{Key-Words}

Consultative power, neighborhood councils, democraty.

Philippe Breton est Professeur des Universités au Centre universitaire d'enseignement du journalisme, Université de Strasbourg, et directeur adjoint de l'unité CNRS, « Cultures et sociétés en Europe ».

Célia Gissinger est doctorante au sein de l'unité CNRS, «Cultures et sociétés en Europe », Université de Strasbourg. 


\section{Les conseils de quartier, un révélateur des difficultés d'émergence du nouveau « pouvoir consultatif » \\ Philippe Breton, Célia Gissinger}

phbreton@club-internet.fr

celia.gissinger@misha.fr

L'une des caractéristiques majeures de l'évolution des sociétés démocratiques européennes dans les décennies 90 et 2000, est l'émergence, au côté des pouvoirs législatif, exécutif, judiciaire, d'un quatrième pouvoir (cinquième si on veut ajouter le "pouvoir médiatique »), le " pouvoir consultatif ». Les « conseils de quartier», tels que la loi de 2002 les dispose en France, constituent l'une des institutions-phare témoignant de la poussée de cette exigence démocratique et de ses difficultés.

Cet article aborde successivement l'historique et les enjeux de ce nouveau pouvoir consultatif, la question des compétences citoyennes à participer à de tels dispositifs, les blocages et les résistances, notamment politiques et associatives, qui obèrent leur déploiement. La question de la séparation des pouvoirs sera au centre de cette analyse qui tente de montrer que le nouveau pouvoir consultatif, est à la fois démocratiquement nécessaire, et techniquement possible, est politiquement difficile à mettre en place. Une enquête de terrain conduite à Strasbourg dans ce domaine apporte une illustration de ces difficultés.

Cette enquête a permis d'observer tout le processus d'élaboration des Conseil de quartier à Strasbourg, prenant en compte aussi bien les enjeux de la mise en place d'un pouvoir consultatif, l'histoire des Conseils de quartier à Strasbourg et en France, ainsi que le contexte politique. Un travail d'archive a donc été effectué sur les Conseils de quartier en France et sur les expériences de pouvoir consultatif en Europe (Gissinger, Breton, 2008).

L'émergence du nouveau pouvoir consultatif: une nécessité démocratique

La démocratie est un régime politique qui peut se définir de différentes façons. Idéalement, ce «régime de la parole» comme le décrit l'historien Jean-Pierre Vernant, se constitue comme une alternative à la tyrannie, donc à la concentration et à la confusion des 
pouvoirs. L'un des principes fondateurs de toutes les sociétés démocratiques, souvent au centre de leurs Constitutions, est le principe de séparation des pouvoirs, comme antidote majeur à la tyrannie. Les dictatures modernes, le nazisme par exemple, se sont imposées en réinstaurant la confusion et la concentration des pouvoirs législatif, exécutif et judiciaire.

\section{L'impératif consultatif}

Les sociétés démocratiques modernes ont vu émerger, comme produit de cette dynamique de séparation des pouvoirs, différents dispositifs, qui tendent à s'institutionnaliser. C'est le cas de ce que nous appelons ici le «pouvoir consultatif». Les institutions qui en découlent, comme les conseils de quartier, ne sont donc pas le produit du hasard ou d'une contingence politique à court terme. Ils ressortent d'abord d'une logique propre au mouvement de séparation des pouvoirs qui caractérise le régime démocratique.

Au-delà de cette dynamique, on constate d'importantes mutations sociologiques, notamment depuis la période d'après-guerre, qui vont dans le sens d'un désir plus grand d'une partie de la population d'être consultée sur les décisions qui la concerne.

Ce phénomène est lié à l'émergence d'individus plus autonomes, à l'accroissement des possibilités d'accéder à l'information et à une forte élévation du niveau culturel et scolaire. Entre également en ligne de compte l'accroissement de la population des retraités actifs et une prise de participation plus active des femmes et des jeunes à la vie sociale, le tout couplé avec la modernisation des pratiques professionnelles et des modes de management de la décision.

Même s'il faut bien, dans ce contexte, prendre la mesure des phénomènes de "sécession sociale » ou, pour parler comme Albert Otto Hirschman, de « défection » (Hirschman, 1995), il n'en reste pas moins qu'une fraction très importante de la population, et notamment dans les grandes villes, qui concentrent pour des raisons historiques et en fonction des mutations foncières actuelles, une part importante des couches moyennes modernistes, est directement concernée par ce phénomène.

À ce facteur il faut ajouter d'une part les mutations qui apparaissent dans les conditions d'exercice du pouvoir comme la complexité et la technicité croissante des décisions et d'autre part l'exigence d'une légitimité mieux assurée de ces décisions auprès des administrés. 
Du point de vue du mécanisme de la décision publique, chacune de ces mutations peut être regardée comme concourant à la fois à la possibilité de participer au mécanisme général de la décision publique et à l'accroissement du désir de participer à son élaboration et à son évaluation. Cette poussée du pouvoir consultatif est donc clairement ressentie comme une véritable nécessité démocratique.

\section{Une institutionnalisation progressive}

Ce thème apparaît dans la littérature de recherche notamment chez Marie-Hélène Bacqué, Henri Rey et Yves Sintomer. Ces auteurs évoquent "l'émergence embryonnaire d'un quatrième pouvoir, celui des citoyens lorsqu'ils participent à la prise de décision, directement (en assemblée générale ou à travers des référendums), à travers des petits groupes tirés au sort (jurys berlinois), ou à travers des délégués étroitement contrôlés (budgets participatifs, structures de développement communautaire) » (Bacqué, Rey, Sintomer, 2005 : 36$37)$.

Par ailleurs de très nombreux débats de société portent la trace de l'exigence citoyenne de s'intégrer à l'architecture politique et démocratique au-delà du simple moment électoral. Les premiers dispositifs de démocratie locale portent la trace de l'existence de ce nouveau pouvoir démocratique.

Certaines de ces structures sont anciennes, les premiers « comités de quartier » sont créés en 1920 à Grenoble ou à Marseille, puis dans les années 50 dans plusieurs grandes villes (Lyon, Rennes...), puis dans la décennie suivante, dans le cadre des structures nées des «luttes urbaines » (la naissance des Groupes d'action municipale à Grenoble).

La loi d'orientation du 6 février 1992 relative à l'administration territoriale de la République constitue une des premières prises en compte institutionnelles de ce nouveau phénomène. Les travaux du Conseil national des villes, notamment, en mai 2001 lors de la «Rencontre nationale des conseils de quartier et de la démocratie locale » (Conseil national des villes, 2001), prolongent la dynamique de ce nouveau "pouvoir consultatif», qui se traduit par la mise en place de nombreux «conseils consultatifs» et de "comités de quartier $»$.

Les deux notions centrales qui servent à penser, à l'époque, cette poussée du pouvoir consultatif, sont celles de «démocratie de proximité » et de «démocratie participative». La tradition philosophique anglo-saxonne ajoutera à cela, dans son propre espace 
culturel, la notion de « démocratie délibérative », à partir des travaux théoriques de Jürgen Habermas et de John Rawls.

Il faut souligner que la poussée de cet « impératif consultatif » est un phénomène de dimension européenne, voire occidentale, avec par exemple, une réflexion très poussée en Allemagne, au Canada ou en Espagne sur les « jurys citoyens » (Daniel, 1997 ; Blanco, 2001).

En France, la loi de 2002 va constituer une étape essentielle dans l'institution de ce nouveau pouvoir consultatif. Elle dispose que chaque commune de plus de 80000 habitants «est dotée d'un conseil de quartier dont le conseil municipal fixe la dénomination, la composition et les modalités de fonctionnement. Les conseils de quartier peuvent être consultés par le maire et peuvent lui faire des propositions sur toute question concernant le quartier ou la ville. Le maire peut les associer à l'élaboration, à la mise en ouvre et à l'évaluation des actions intéressant le quartier, en particulier celles menées au titre de la politique de la ville. Le conseil municipal peut affecter aux conseils de quartier un local et leur allouer chaque année des crédits pour leur fonctionnement. ${ }^{124}{ }^{\prime}$

\section{Le « pouvoir consultatif » est techniquement possible.}

Ainsi que nous l'avons observé, notamment sur le terrain de la Ville de Strasbourg, l'absence de compétence citoyenne est une objection qui apparaît fréquemment dans les mises en œuvre du pouvoir consultatif, et notamment dans les conseils de quartier. Pourtant, de nombreuses expériences ont montré que, malgré les disparités, par exemple culturelle ou scolaire, entre citoyens, il est possible qu'ils produisent un avis raisonné et représentatif.

\section{Tirage au sort et compétence citoyenne :}

La compétence citoyenne peut se définir selon trois éléments: "d'abord, la capacité à se former une opinion, librement, sur un sujet donné ; ensuite, celle d'argumenter à égalité ces opinions, c'est-àdire de convaincre sans violence et sans ruse, dans le cadre d'une conflictualité pacifié ; enfin, de combiner l'écoute et la prise de parole dans une démarche d' "empathie cognitive ».» (Breton, $2006: 21)$. La longue expérience des jurys populaires en procès d'assises, comme forme de «pouvoir consultatif » effectif, issue d'une première vague de séparation des pouvoirs en Grèce antique, montre que l'appel à la compétence citoyenne est techniquement possible. Cette instance a

${ }^{124}$ Loi n ${ }^{\circ} 2002-276$ du 27 février 2002 relative à la démocratie de proximité, Art. L. 2143-1 
connu de nombreuses réformes en France depuis sa mise en place en 1790 afin d'élargir le tirage au sort des jurés à des couches de plus en plus larges de la population. Leur objectif était de rendre le jury populaire le plus représentatif possible.

Comme l'explique Mogens Herman Hansen, au temps de la démocratie athénienne, le tirage au sort ne consistait pas à considérer "que tous les hommes fussent égaux en compétence, mais qu'ils étaient tous assez compétents pour ce qu'on leur demandait» (Hansen, 1993 : 175). Permettre à tout citoyen de participer à une décision de justice, ou lui demander son avis dans le cadre de conseil de quartier, c'est donc faire appel au citoyen dans son rôle d' ' autorité publique » (Rey, $2005:$ 1715), doté en tant que tel d'une compétence appropriée.

Afin de permettre à tout citoyen d'exercer une compétence citoyenne, un dispositif de pouvoir consultatif doit donc être accompagné d'un tirage au sort. L'histoire de la démocratie nous fournit de nombreux exemples où la question de la représentativité est réglée par cette procédure. Comme le dit Yves Sintomer « contre l'idée rabâchée que les citoyens ordinaires sont incompétents et que le recours au tirage au sort aboutit à désigner des médiocres et des incultes incapables d'orienter la cité de façon sensée, l'expérience montre qu'une participation organisée de façon délibérative n'est pas seulement démocratique mais qu'elle aboutit à des résultats raisonnables " (Sintomer, 2007 : 140)

La question de la compétence du citoyen est donc moins de savoir si le citoyen est capable ou pas, que de se demander comment le citoyen est informé des dossiers pour lesquels on lui demande son avis. La question du déroulement des débats ne peut donc être évitée.

\section{Animation et procédure des débats :}

Le constat que l'on peut faire, sur le terrain des conseils de quartier, à partir d'un travail d'archives et d'observations faites sur le fonctionnement des conseils de quartier à Strasbourg depuis leur création jusqu'en 2007, est que les citoyens qui participent à ces instances aux côtés des associations et des élus de quartier ont bien peu de place pour exprimer leurs avis. Ce constat recoupe l'analyse des « dérives » que pointent plusieurs chercheurs ayant travaillé sur le même sujet, comme George Gontcharoff, qui note les dérives associées à « la volonté organisatrice du pouvoir local » (Gontcharoff, 1999 : 305) ou Catherine Neveu. Cette dernière pointe par exemple «la large absence d'autonomie constatée, produit d'une part des 
processus d'invisibilisation de l'action collective, de ritualisation dans les relations entre Comités et Mairie et autres institutions; et d'autre part des processus de repli sur soi, voire de "dérive ethnique » ( Neveu, $2003: 224$ )

A l'image des assemblées générales, l'absence d'un animateur neutre et indépendant transforme la parole en un rapport de force entre participants. L'observation sur le terrain de la Ville de Strasbourg, à la période considérée, montre que la fonction d'animateur est généralement occupée par un élu de la majorité municipale (Breton, Gissinger, 2008).

Or, le bon déroulement d'un débat qui permet à chacun d'exercer ses propres compétences ou connaissances est techniquement possible. L'exemple des «jurys citoyens » mérite d'être cité en raison de leur réussite technique comme dans le cas des «plannugszelle» initiés par le professeur Peter Dienel et celui des «conseils citoyens» mis en place en Espagne. Leur but est de créer temporairement un groupe de citoyens à qui l'on présente un projet important pour la ville en lui faisant auditionner les différents techniciens pour lui donner une pleine connaissance du dossier, afin qu'il puisse formuler un avis positif ou négatif. Dans le cas allemand, l'université offre donc, "clef en mains », un dispositif consultatif ponctuel qui produit rapidement un avis consultatif représentatif.

D'après les analyses qui ont été faites sur les «plannugszelle» en Allemagne, la plupart des personnes tirées au sort pour faire partie de ces jurys, "changent d'opinion au cours du processus et se sentent au final compétents pour prendre une décision rationnelle " (Vergne, 2008: 14). Ce phénomène est décrit comme «l'effet d'activation» (empowerment). Mais, comme le dit l'auteur de l'étude, «c'est aux organisateurs de faire jouer leurs talents de modérateurs et de conciliateurs pour monter un programme de travail équilibré tout en restant assez libres vis-à-vis de l'autorité mandataire» (Vergne, $2008: 16)$.

Cette réussite technique démontre que les participants, à l'inverse de ce que l'on pourrait craindre, font abstraction de leurs intérêts particuliers et " tendent à faire passer le long terme et l'intérêt général au premier plan » (Vergne, $2008: 9$ ).

Les conditions qui permettent la réussite technique de ces «jurys citoyens » sont, outre le tirage au sort que nous avons évoqué plus haut, une procédure de débat standardisée, l'animation des débats par un tiers neutre, n'intervenant pas sur le contenu mais sur la procédure 
de débat et une finalité consultative claire à l'esprit de tous les participants.

La question de l'animation des débats est une problématique prise en charge par les sciences de l'information et de la communication, notamment lorsque celles-ci s'intéressent, dans la tradition habermassienne et, in fine, aristotélicienne, au fonctionnement des débats argumentés et à leur rôle dans la formation d'une opinion publique démocratique (Breton 2006 et 2008).

\section{Les résistances à la poussée du pouvoir consultatif}

Comme nous venons de le voir, la poussée démocratique en faveur de nouvelles instances consultatives est bien réelle et les expériences réalisées dans ce domaine prouvent qu'il n'y a guère d'obstacles à leur développement du point de vue des "compétences citoyennes ", à condition, bien sûr, que certaines conditions soient réalisées.

Une condition essentielle à la mise en place de ces instances est la mise en œuvre du principe de séparation des pouvoirs. Ceci implique que les instances de consultation soient détachées du politique et, d'une façon générale des intérêts particuliers. Là réside la principale source de blocage au déploiement, notamment de conseils de quartier, qui répondent aux normes de la nouvelle exigence démocratique.

\section{Un bilan globalement négatif}

Tout devrait concourir donc, à un formidable développement de ce point de vue. Pourtant le bilan que fait par exemple Loïc Blondiaux est négatif. Ce dernier diagnostique que «dans la pratique, la plupart des expériences mises en cuvre aujourd'hui en France placent les habitants auxquels ils s'adressent dans une série de doubles contraintes qui, au final, justifient leurs déceptions et alimentent leurs soupçons envers ce type de démarche. On leur demande ainsi de s'exprimer mais, dès qu'ils le font un peu trop fort, la parole leur est retirée. [...] On comprend mieux ainsi pourquoi la plupart des expériences qui cherchent à institutionnaliser la participation des " simples citoyens » dans la durée échouent faute de combattants, par désertion pure et simple de leur publi. »(Blondiaux, 2000 : 130).

Les critiques portent sur l'absence de séparation des pouvoirs entre l'exécutif et le législatif et ce que cela induit du point de vue de la confusion de l'intérêt général et des intérêts particuliers, ainsi que du peu de représentativité des habitants qui participent au conseil de quartier. Il faut remarquer que la mise en place des conseils de quartiers s'accompagne généralement d'une absence d'explicitation de 
la finalité de ces conseils, chaque institution présentant le fonctionnement de ses conseils de quartier comme le plus «naturel» ou le plus «évident», en les laissant se noyer dans des processus de « ritualisation» (comme le remarque Catherine Neveu, qui souligne que « cette ritualisation serait donc ce qui permet d'exclure du champ politique la question de la participation »(Neveu, 2003 : 120), ou plus généralement, en évitant tout simplement la question.

Aussi ambitieuses que soient les volontés pour rendre plus démocratiques les conseils de quartier, leurs modalités concrètes d'application font apparaître des blocages qui peuvent remettre en question ces ambitions. Sur le terrain des conseils de quartier, on peut constater la résistance dans de nombreuses villes à faire appel aux citoyens «ordinaires" pour composer le collège des habitants. En effet, si certains pensent que l'avis de l'habitant est important parce qu'il connaît son quartier, d'autres avancent l'idée que tous les habitants n'ont pas le même engagement dans la vie de leur quartier et qu'il vaut mieux recourir à des listes de volontaires pour avoir les plus aptes à la participation citoyenne.

Cependant, considérer un citoyen plus compétent qu'un autre pour participer à la vie politique risquerait de faire de ces volontaires des « citoyens professionnels », monopolisant la participation citoyenne dans les conseils de quartier. Cette professionnalisation a été concrètement observée chez les anciens participants des Conseils de quartier à Strasbourg.

\section{L'analyse d'un échec}

Comment peut-on, en conclusion, analyser cet échec? Pour comprendre cette situation, il faut sans doute suivre la piste de ce qui constitue le noyau dur du nouveau pouvoir consultatif. Celui-ci s'appuie sur deux principes dont la mise en œuvre apparaît nécessaire pour qu'il puisse véritablement se déployer, au sein des conseils de quartier, mais plus généralement dans toute instance qui se veut « consultative ».

Le premier principe est celui de la séparation des pouvoirs. Nous sommes là au cœur de la dynamique démocratique qui pousse précisément à l'institutionnalisation du consultatif. Le second principe est celui de l' "intérêt général », lui aussi au cœur de l'invention démocratique, qui tente d'articuler, en donnant la prééminence au premier niveau, intérêt général et intérêts particuliers.

L'application de ces deux principes implique une double rupture. Le pouvoir politique, notamment municipal dans le cas des conseils de 
quartier, doit se dessaisir d'une partie de ses prérogatives. Dans la plupart des villes, les conseils de quartier sont traditionnellement dirigés ou "présidés » par des élus. Ce sont eux qui fixent les ordres du jour. Ces structures sont sous la tutelle du politique. Appliquer le principe de séparation des pouvoirs nécessiterait donc que les conseils de quartier gèrent eux-mêmes leur propre fonctionnement et que les élus soient en dehors de leur périmètre d'action. Comme nous l'avons vu dans le cas des plannungzelle, la réussite du dispositif tient à ce que la consultation est pilotée par un animateur neutre.

Très peu de conseils municipaux, quelle qu'ait été leur audace pendant la campagne électorale et malgré des politiques d'affichage très offensives sur le thème de la "nouvelle gouvernance » par exemple, acceptent de se dessaisir concrètement de cette prérogative, qui constitue souvent une part importante du pouvoir qu'exercent les élus sur le terrain. Le paradoxe est clair, c'est ceux qui ont le pouvoir qui doivent le partager...

Appliquer l'autre principe qui est d'articuler la fonction consultative à une finalité d'intérêt général, pose également de redoutables problèmes. Les conseils de quartier sont un lieu traditionnel où le pouvoir réel se négocie entre les élus et les représentants d'association ou de groupements privés (représentant les commerçants par exemple). Ce face à face au sein des conseils de quartier (dont les habitants se sentent souvent exclus) favorise peu l'émergence d'un réel pouvoir consultatif.

Les conseils de quartier, pour répondre aux normes du nouveau pouvoir consultatif, doivent tenir les associations à l'écart de leur périmètre pour privilégier des groupes d'habitants tirés au sort, travaillant dans des dispositifs de parole qui garantissent la prise en compte de l'intérêt collectif. Les associations, outre le fait que cellesci représentent, par nature, des intérêts toujours particuliers, sont souvent un lieu investi par le politique.

La double pression des élus peu enclins à se dessaisir de leurs prérogatives et des représentants du monde associatif qui veulent à tout prix maintenir leurs positions - souvent chèrement acquises dans des structures proches du pouvoir, conduit dans les faits à ce que la plupart des conseils de quartier des grandes villes françaises, soient vidées de leur potentialité à être le siège d'un véritable pouvoir consultatif. Ces freins mis à la dynamique du «big-bang» démocratique risquent évidemment de créer des frustrations importantes dans l'opinion publique, frustrations dont les 
Dossier : Repenser la communication des organisations publiques

conséquences politiques risquent à terme de menacer la démocratie elle-même.

\section{Bibliographie}

BACQUE M.-H., REY H., SINTOMER Y., Gestion de proximité et démocratie participative. Une perspective comparative. Paris: La découverte, 2005, $314 \mathrm{p}$.

BLANCO I., Les jurys citoyens en Espagne : vers un nouveau modèle de démocratie locale ?, Texte traduit de l'espagnol par GIRAUD V. Mouvements, Universitat autónoma de Barcelona. 2001, $\mathrm{N}^{\circ} 18$, pp.132-137.

BLONDIAUX L., «La démocratie par le bas, Prise de parole et délibération dans les conseils de quartier du vingtième arrondissement de Paris », In Hermès, 2000, n²6-27, pp. 323-338.

BRETON Ph., L'incompétence démocratique. La crise de la parole aux sources du malaise (dans la) politique, Paris: La découverte, 2006, $262 \mathrm{p}$.

BRETON Ph., Convaincre sans manipuler, Paris: La découverte, 2008, 154 p.

Conseil National des Villes: rencontre nationale des conseils de quartier et de la démocratie locale [en ligne] : Mai 2001. Disponible sur: $\quad<$ http://www.ville.gouv.fr/infos/cnv/avis/quartiers.html $>$ (consulté le 28.10.2008)

DIENEL P., Die Plannungszelle : der burger plant seine umwelt. Eine alternative zur establishment-demokratie, Opladen: Westdeutscher Verlag, 1997.

GISSINGER C., BRETON Ph., Quelle architecture pour les conseils de quartier dans le contexte d'un nouveau pouvoir consultatif ? [en ligne] : 2008. Disponible sur :

http://argumentation.blog.lemonde.fr/2008/07/01/quelle-architecturepour-les-conseils-de-quartier-dans-le-contexte-d\%E2\%80\%99unnouveau-pouvoir-consultatif-premiere-partie-la-loi-de-2002-et1\%E2\%80\%99emergence-du-pouvoir-consultatif/

http://argumentation.blog.lemonde.fr/2008/07/03/conseils-de-quartier$\underline{2 />}$

GONTCHAROFF G., Le renouveau des comités de quartier, in La démocratie locale. Représentation, participation et espaces publics, Curapp-Craps, PUF, 1999, p. 305-329 
Les conseils de quartier...

HANSEN M. H., La démocratie athénienne. A l'époque de Démosthène, Paris : Les belles lettres, 1993, 493 p.

HIRSCHMAN A. O., Défection et prise de parole, Paris : Fayard, 1995, $212 \mathrm{p}$.

NEVEU C., Citoyenneté et espace public. Habitants, jeunes et citoyens dans une ville du Nord, Presses universitaires Septentrion, 2003, 246 p.

REY A., Dictionnaire culturel de la langue française, Le Robert, 2005, 9600 p (4 volumes de 2400 p.).

SINTOMER Y., Le pouvoir au peuple. Jurys citoyens, tirage au sort et démocratie participative, Paris : la Découverte, 2007, 177 p.

VERGNE A., Les jurys citoyens : une nouvelle chance pour la démocratie? Mode d'emploi et bilan de trente ans de pratique du modèle "Planungszelle" en Allemagne, Les notes de la fondation Jean-Jaurès, 2008, n 12,33 p. 